\title{
Biomechanical Analysis of Postural Sway in Elderly Adults on Ramps
}

\author{
John J. Eichman and Randa L. Shehab \\ School of Industrial Engineering \\ University of Oklahoma \\ Norman, Oklahoma
}

\begin{abstract}
This study investigated the effects of ramp angles on postural deviation as a function of age. Five ramp inclinations $(1: 8,1: 10,1: 12,1: 16$, and 1:20) were examined in both ascent and descent directions. Five younger ( 22 to 28 years) and five older (78 to 88 years) adults participated in the study. Video-based motion analysis was used to measure torso and hip angles while participants walked on an adjustable inclined ramp. Both young and older participants had a significant increase in torso angle across ramp slopes from ascent to descent. In addition, the data indicated that older participants tended to lean to the right while walking while the young participants leaned to the left. Measurements of hip angle revealed that young participants had significantly greater hip movement than older participants and that hip angle decreased significantly as participants transitioned from descent to ascent trials. Based on the data observed, it is possible that ramp descent is more problematic for elderly adults. However, within the ramp conditions evaluated, the data were unable to clearly discriminate between ramp slopes beyond identifying differences between slopes of ascent and descent.
\end{abstract}

\section{INTRODUCTION}

As the 21 st century has come, there is a need to refine experiments to consider elderly adults. According to Howell (1997), nearly $13 \%$ of the population is at an age of retirement (i.e., 65 and older). One area of importance for the elderly population is building access. Access to public buildings is important because it allows the older population to maintain independence which promotes a higher quality of life. If a building entrance is not at street level, access can be provided in two forms, stairs and ramps. However, stairs could cause problems for the older adult. For example, the relative loss of muscle strength in older adults (Vercruyssen, 1997) might increase their risk of tripping or losing balance on stairs, which consequently increases their risk of falling. One alternative to stairs is a ramp. Ramps are used, not only as a means of access in and out of public buildings, but also to provide assistance in transferring between levels within buildings. Single-story buildings with multiple elevations typically provide both stairs and ramps. However, the ramp guidelines supported by the Americans with Disabilities Act Accessibility Guidelines (ADAAG) were designed primarily for people bound to wheelchairs and may not include the ambulatory elderly population.

Two processes that are the foundation of independent mobility are the control of balance and the ability to integrate balance adjustments into ongoing voluntary movements such as reaching for or lifting objects (Woollacott, 1993).

Secondary to disease processes associated with aging or to the aging process itself, balance often becomes impaired as one grows older (Hughes, Duncan, Rose, Chandler, and Studenski, 1996). Numerous authors have defined postural sway as the ability to maintain one's balance. Hageman, Leibowitz, and Blanke (1995) defined postural control as the ability to maintain the body's center-of-gravity over the base of support during quiet standing and movement. The ability to maintain postural control is critical for successful performance of nearly every daily task.

Falling is one of the most serious problems facing older adults. Falls are the leading cause of accidental death in the over 65 years age group (Maki, Holliday, and Fernie, 1990). Fear of falling leads to a loss of confidence and reduced activity, resulting ultimately in a loss of independence (Maki, Holliday, and Topper, 1991). Although falling is a complex and multifactorial problem, there is little doubt that deterioration in postural balance is one of the major contributing factors (Maki et al., 1990). The decrease in information from kinesthetic receptors or decrease in the use of that information in the central nervous system may also contribute to the higher incidence of falls (Kroemer, 1997). Safe building access is necessary to minimize the risk of falls.

\section{PROBLEM DEFINITION}

The research presented in this paper addresses the issue of older adults and their ability to walk on ramps of various inclinations. Wolfinbarger (2000) stated that it may be safer to walk on ramps rather than stairs because walking on ramps is more closely related to walking on a flat surface. Consequently, the use of ramps may reduce the risk of trips and missteps (Jackson and Cohen, 1995). The current study investigated relationships between postural sway and ramp slope. The current ADA guideline of ramp slope (1:12, one foot of rise for every 12 feet of run) was examined along with four other ramp slopes. The goal of this study was to identify appropriate ramp slopes for older adults. This was achieved by investigating multiple slopes to identify if a critical slope exists at which balance on ramps becomes impaired. 


\section{METHODS}

\section{Participants}

Forty-three participants were recruited from local churches, University of Oklahoma faculty and students, retirement homes, and teaching seminars. Participation in the experiment was a two-step process. First, all elderly participants completed a questionnaire, to determine preferences and patterns of use for ramps or stairs (Wolfinbarger and Shehab, 2000). Second, survey participants who volunteered for the experiment were selected based on qualification into two age groups. Six participants were classified as young (average age $=24.6$ years) and six participants were classified as older (average age $=81.8$ years).

\section{Equipment}

An adjustable wooden ramp was used as the test instrument. The ramp was $6 \mathrm{ft}$ wide and $16 \mathrm{ft}$ long with an 8 $\mathrm{ft}$ inclined section. Several safety features were incorporated: 1) handrails around one side and both ends, 2) padded mats on the floor along the open edge of the ramp, 3) a bright yellow 12 -inch wide strip along the open edge of the ramp, and 4) a texturized non-slip additive in the painted surface. In addition to providing better frictional characteristics, the non-slip additive helped simulate the feel of a concrete, exterior ramp. A video based motion analysis system was used to capture data. Two cameras provided views of motion in the sagittal plane and in the frontal plane. The video captured movement through the use of reflective markers. Markers were located at the C7, the right and left tip of the scapula, the L5/S1, and the right and left posterior center of hip rotation, following guidelines proposed in Chaffin and Anderson (1984). PEAK Motus (version 4.3.1) software was used to digitize the video and calculate characteristics of motion. When analyzed, the markers produced the stick figure as shown in Figure 1.

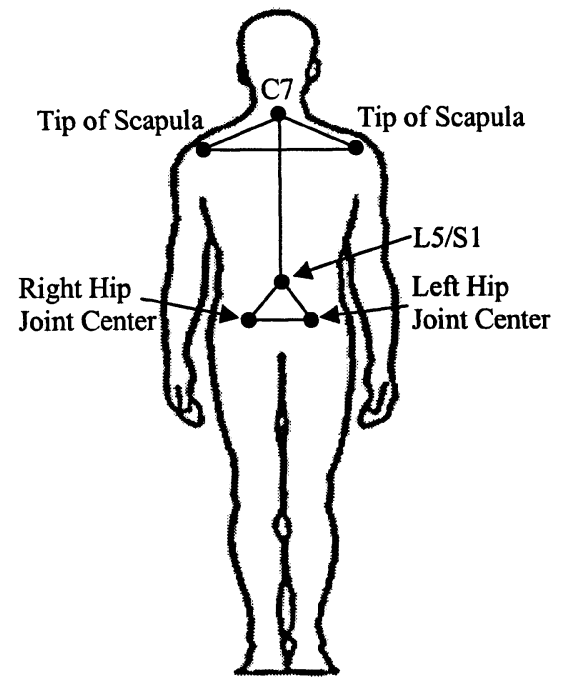

Figure 1. Stick Figure Defined by Marker Location.

\section{Experimental Design}

The study used a repeated measures design with ramp as a within subject effect and age as a between subjects effect. Five ramp slopes were evaluated: 1)1:20 $\left(2.9^{\circ}\right)$, 2) 1:16 $\left(3.6^{\circ}\right)$, 3) $1: 12\left(4.7^{\circ}\right)$, 4) $1: 10\left(5.7^{\circ}\right)$, and 5) $1: 8\left(7.1^{\circ}\right)$ in both ascent and descent directions of travel. These ten combinations were examined along with a baseline or flat slope, creating 11 unique ramp conditions. Performance was evaluated using measures of torso angle (TA) and hip angle (HA). TA represented leaning of the torso to one side of the vertical axis and was measured as the angle created by the $C 7$ vertebra, the lumbscacral center at the L5/S1 (vertex), and the y-axis. HA represented the tilt in the pelvis about the horizontal axis and was measured by the angle created between the right posterior hip joint center, left posterior hip joint center, and the $\mathrm{x}$-axis.

\section{Task Procedure}

Before the experiment, all participants signed an informed consent form. The older participants were administered the Mini-Mental State Examination (MMSE) to screen for evidence of dementia (which would have lead to removal from the study). Participants changed into the experimental clothing and were instrumented with reflective markers for video recording.

Participants began by familiarizing themselves walking on the ramp at an angle of 1:12 (ADA guideline). The experiment began with the baseline trial (flat slope). Due to the difficulty in changing the direction of incline of the ramp, all slopes were completed in succession for a given ramp direction. For each participant, the order of the ramp condition was counterbalanced to first determine direction of travel and then to order the slopes within each direction. Participants performed three trials at each experimental condition and were given ample opportunity to rest between trials.

\section{RESULTS AND ANALYSES}

To characterize postural sway during gait, three measures of torso angle were assessed: 1) maximum torso angle to the left of vertical (TAmax-L), 2) maximum torso angle to the right of vertical (TAmax-R), and 3) overall maximum torso angle (TAmax) regardless of the direction of the deviation. Measures of torso angle can be interpreted as surrogate measures of postural instability in that the larger the torso angle, the further the center of gravity is removed from the base of support. Hip angle was also assessed using a single measure of overall maximum hip angle (HAmax). This value may have occurred either above or below the horizontal axis and was primarily used to determine if any noted torso motion could be accounted for by a larger whole-body movement that may also have been evident in hip motion. In other words, hip angle was used to investigate the extent of the torso motion 
and determine if it was contained entirely in the torso or was it part of a larger body movement.

Table 1 summarizes the results of the analyses of variance with a presentation of the significant effects for each measure. Note that subject (nested within age and gender) and the interaction of ramp and subject were significant in all analyses. This result is typical and can be attributed to the large individual differences between participants with regard to their gait speed, stride length, and movement patterns. The ramp by subject interaction indicates that these individual differences were also evident as differences in the manner by which the eleven ramp slopes affected participants.

The main effect of ramp was significant for both TAmax$\mathrm{R}$ and Hamax. A closer examination of TAmax-R (as shown in Figure 2) suggests that as ramp inclination progressed from a steep ramp descent, through a flat inclination, and then through a steep ramp ascent, TAmax- $R$ tended to increase slightly. This trend is more apparent for the older participants whose values of TAmax-R showed a rather steady but small increase and were substantially larger than that of the younger participants. Younger participants' Tamax-R seemed to increase slightly as the descent became less steep but remained fairly steady across the base and ascent ramp slopes.

Table 1. Summary of Significant Effects.

\begin{tabular}{|l|l|c|c|c|}
\hline Variable & df & MS & F & $p$ \\
\hline \multicolumn{5}{|c|}{ TAmax } \\
\hline $\mathrm{S}(\mathrm{AG})$ & 6 & 25.52 & 37.28 & $<.0001$ \\
\hline $\mathrm{R} \times \mathrm{S}(\mathrm{AG})$ & 60 & 1.61 & 2.35 & $<.0001$ \\
\hline \multicolumn{5}{|c|}{ TAmax-L } \\
\hline $\mathrm{S}(\mathrm{AG})$ & 6 & 42.54 & 54.75 & $<.0001$ \\
\hline $\mathrm{R} \times \mathrm{S}(\mathrm{AG})$ & 60 & 1.53 & 1.97 & .0010 \\
\hline \multicolumn{5}{|c|}{ TAmax-R } \\
\hline $\mathrm{R}$ & 10 & 3.35 & 2.96 & .0043 \\
\hline $\mathrm{S}(\mathrm{AG})$ & 6 & 21.61 & 31.0 & $<.0001$ \\
\hline $\mathrm{R} \times \mathrm{S}(\mathrm{AG})$ & 60 & 1.13 & 1.62 & .0144 \\
\hline \multicolumn{5}{|c|}{ HAmax } \\
\hline $\mathrm{R}$ & 10 & 15.00 & 9.36 & $<.0001$ \\
\hline $\mathrm{A}$ & 1 & 1116.42 & 11.27 & .0153 \\
\hline $\mathrm{S}(\mathrm{AG})$ & 6 & 99.06 & 263.21 & $<.0001$ \\
\hline $\mathrm{R} \times \mathrm{S}(\mathrm{AG})$ & 60 & 1.60 & 4.26 & $<.0001$ \\
\hline
\end{tabular}

HAmax also exhibited a significant ramp effect that can be seen in Figure 3. The trend evident for HAmax is opposite that described for TAmax-R. As ramp slope progressed from a steep ramp descent to a steep ramp ascent, the maximum hip angle tended to decrease. This trend was strongest for the young participants whose HAmax decreased by almost $4^{\circ}$. In addition, the data indicated that the young participants had rather large hip motions across all trials. Older participants tended to minimize their hip motion much more than the young participants and only exhibited a small decrease in motion (a change of less than $2^{\circ}$ ) from the descent to the ascent trials. Older participants' hip movement remained fairly steady across the descent and base trials as well as across the ascent trials.

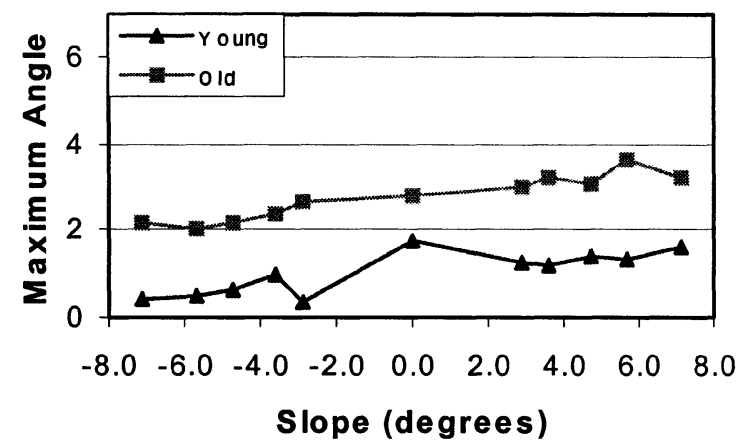

Figure 2. Maximum Torso Angle-Right by Slope for Young and Older Participants.

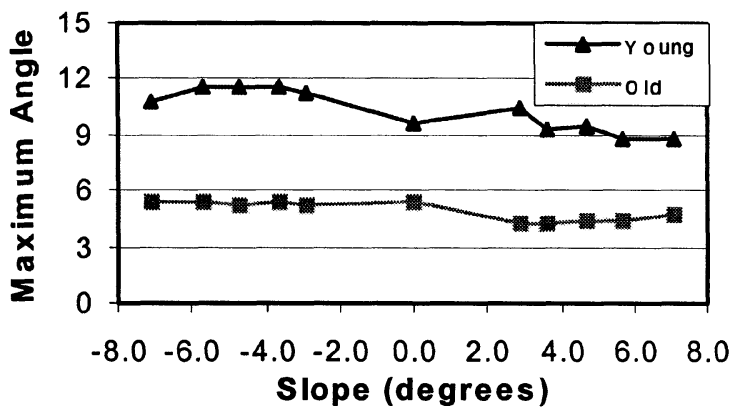

Figure 3. Maximum Hip Angle by Slope for Young and Older Participants.

Although Figure 2 appears to show a distinct difference between age groups, age was not significant $(p=0.0000)$ for any measure of torso angle. The large variability between participants and within age groups statistically prevented age from reaching significance. However, an interesting and apparently age-related phenomenon was noted in the torso angle data. Older participants consistently exhibited higher values of TAmax-R while younger participants consistently exhibited higher values of TAmax-L (see Figure 4). Although no biomechanical or functional explanation could be determined, the handrail was always located to the right of the direction of travel and it was surmised that perhaps older participants were unconsciously leaning toward the handrail. 


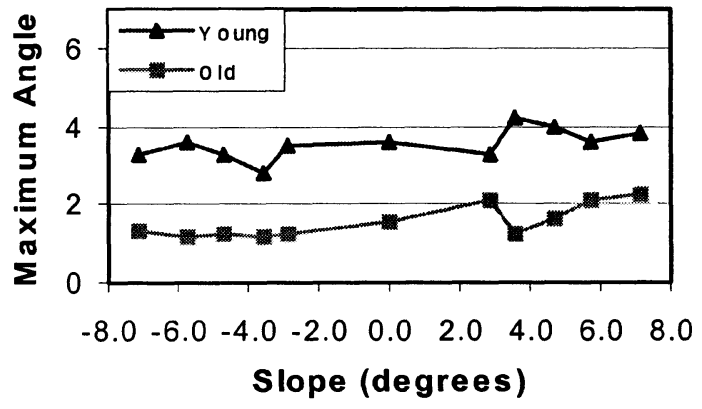

Figure 4. Maximum Torso Angle-Left by Slope for Young and Older Participants.

The only measure in which the factor age attained significance was HAmax. As shown in Figure 3, the younger participants had consistently higher values of hip angle across all trials. Initially, it was expected that older participants would exhibit the larger hip angles due to reduced muscle strength and control capabilities. However, the data suggest that older participants may have compensated for these muscular reductions be walking in a much more controlled manor than did the young participants.

One effect of interest, but that was not significant for any of the measures, was the ramp by age interaction. It was initially hypothesized that an inclination would have a differential effect on age groups, with a larger negative impact on elderly performance. However, given the magnification of individual differences and capabilities within the elderly population, it was not surprising that the variability between participants (both within and across age groups) was too overwhelming.

\section{DISCUSSION}

The results of the present study revealed that slope did have some effect on measures of postural sway during a ramp walking task. However, there were no clear trends among the different ramp slopes. In addition, the effects of age group were inconsistent.

The original intent of this study was to examine ramp slopes in order to determine if a critical slope exists at which there is an increase in postural instability. Examination of the results for torso angle indicated that for both age groups, traveling down a ramp was not particularly problematic from the viewpoint of creating a larger instability due to increased torso movement. However, travel up a ramp did tend to create greater movement in the torso and possibly cause greater instability in the upper body. Although ramp slope did attain significance, the results were unable to discriminate between ramp slopes in that all descent trials were similar and all ascent trials were similar. In addition, the ADA guideline was indistinguishable from the other ramp slopes within the same direction of travel.
The measure of hip angle was similarly unable to distinguish between ramp slopes. Although the data showed smaller hip angles for ascent trials, the ramp slopes still tended to group around direction of travel. That is, little difference was evident between descent slopes and little difference was evident between ascent slopes. The trend for larger hip motion during descent trials was probably due to the tendency to use less control when walking downhill. This tendency towards a more "falling" style of gait would tend to suggest that descent trials may cause some level of instability within the pelvic region. Walking uphill typically requires greater muscular control which tends to reduce hip motion. Again, within the range of ramp slopes examined, there was no evidence of any particular slope (including the ADA recommendation of $1: 12$ ) being substantially more problematic than any of the others.

If the data are reviewed from the perspective of overall motion during ramp walking, it is apparent that the general trends hold regardless of participant age. Hip motion is the primary contributor to body motion when walking on ramps. When descending ramps, torso movement tends to play less of a role in overall movement while the influence of hip movement is stronger. This phenomenon of a "falling" gait style may be more problematic for the older adult. Due to the loss of muscle strength and control in the lower extremities, descent may impose a larger risk of falling.

However, when ascending ramps, torso movement tends to contribute more and hip movement tends to provide a smaller contribution. Gait during ramp ascent requires more control which may actually be more physically demanding, particularly for the older adult. However, there is little in the data to suggest that ascent increases the risk of falling.

Although beyond the scope of the current study, an assessment of limits of postural stability would be helpful to understanding the implications of the data. Although the patterns of change were consistent between age groups, it may have been that there was an underlying age-related change in the limits of postural stability such that the older participants were much closer to their stability threshold than the young participants. It would also be interesting to examine the role of age-related visual impairments in ramp walking. Is the reduction of visual perception problematic during ramp walking and does it lead to an increased risk of falling? In addition, it would be valuable to correlate the current results to the natural compensations in stride length and gait speed during ramp walking. The observed postural changes may be caused by other such characteristics of the gait cycle.

Perhaps these additional studies and measures would provide more insight to understanding the stability changes observed in this study. 


\section{REFERENCES}

The Access Board (2001). ADA Accessibility Guidelines for Buildings and Facilities. Internet: www.accessboard.gov/adaag/html/adaag.htm. Accessed on July 16, 2001.

Chaffin, D.B. and Anderson, G. (1984). Occupational Biomechanics. New York: John Wiley \& Sons, Inc.

Hageman, P.A., Leibowitz, J.M., and Blanke, D. (1995). Age and gender effects on postural measures. Archives of Physical Medicine and Rehabilitation, 76, 961-965.

Howell, W.C. (1997), Foreword, Perspectives, and Prospectives. In A.D. Fisk and W.A. Rogers (Eds.), Handbook of Human Factors and the Older Adult (pp. 16). San Diego: Academic Press.

Hughes, M.A., Duncan, P.W., Rose, D. K., Chandler, J.M., and Studenski, S.A. (1996). The relationship of postural sway to sensorimotor function, functional performance, and disability in the elderly. Archives of Physical Medicine and Rehabilitation, 77, 567-572.

Jackson, P.L. and Cohen, H.H. (1995). An in-depth investigation of 40 stairway accidents and the stair safety literature. Journal of Safety Research, 26, 151-159.

Kroemer, K.H.E. (1997). Anthropometry and Biomechanics. In A.D. Fisk and W.A. Rogers (Eds.), Handbook of
Human Factors and the Older Adults (pp. 87-124). San Diego: Academic Press.

Maki, B.E., Holliday, P.J., and Fernie, G.R. (1990). Aging and postural control: A comparison of spontaneous- and induced-sway balance tests. The Journal of American Geriatric Society, 38(1), 1-9.

Maki, B.E., Holliday, P.J., and Topper, A.K. (1991). Fear of falling and postural performance in the elderly. The Journal of Gerontology, 46(4), M123-131.

Vercruyssen, M. (1997). Foreword, Perspectives, and Prospectives. In A.D. Fisk and W.A. Rogers (Eds.), Handbook of Human Factors and the Older Adult (pp. 16). San Diego: Academic Press.

Wolfinbarger, K.G. (2000). The effects of ramp slope of footfloor angle and clearance in elderly adults. Unpublished Thesis, Norman OK: the University of Oklahoma, School of Industrial Engineering.

Wolfinbarger, K.G. and Shehab, R.L. (2000). Stair and ramp design for older adults. In Proceedings of the Human Factors and Ergonomics Society 44th Annual Meeting (CD-Rom). (pp. 4-76 - 4-79) Santa Monica, CA: Human Factors and Ergonomics Society.

Woollacott, M.H. (1993). Age-related changes in posture and movement. The Journal of Gerontology, 48(special issue), 56-60. 